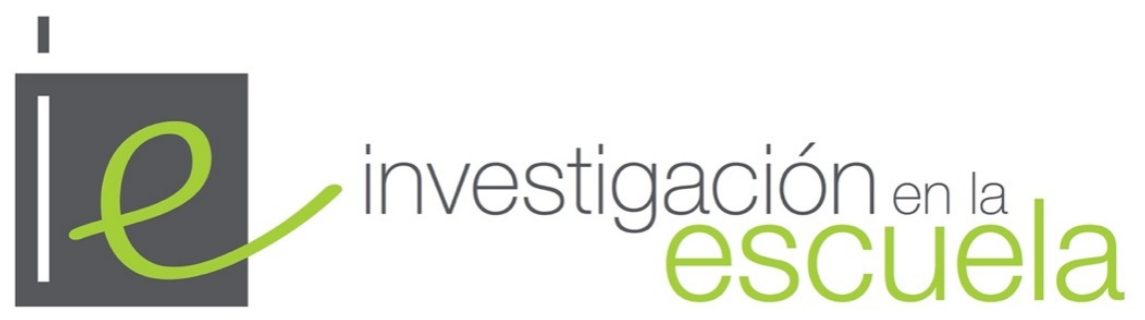

Revista de Investigación e Innovación Educativa nº 103, 2021 | e-ISSN 2443-9991

\title{
La arqueología experimental como estrategia educativa: realidad y posibilidades
}

Experimental archaeology as a learning strategy: reality and possibilities

iD Grancisco José Montoya Martínez es Arqueólogo y egresado de la Facultad de Letras de la Universidad de Murcia (España) franciscojose.montoya@um.es·https://orcid.org/0000-0003-3161-6070

iD Dr. Alejandro Egea Vivancos es Profesor Titular en la Facultad de Educación de la Universidad de Murcia (España)·alexegea@um.es·https://orcid.org/0000-0002-6047-2670

Cómo citar este artículo

Montoya Martínez, F.J. y Egea Vivancos, A. (2021). La arqueología experimental como estrategia educativa: realidad y posibilidades. Investigación en la Escuela, 103, 139-152. doi: http://dx.doi.org/10.12795/IE.2021.i103.10

Resumen. Desde hace décadas en los centros educativos españoles se han multiplicado experiencias didácticas o talleres de arqueología experimental. ¿Aprovechan estas actividades todo su potencial educativo? A través de una metodología de investigación mixta y por medio de un muestreo no probabilístico y no aleatorio se analizan once proyectos de arqueología experimental que se han implementado en los últimos años en las aulas de Educación Secundaria en España. El estudio de estos casos, obtenidos a través de la bibliografía, de la información hallada en Internet y a través de entrevistas con empresas especializadas, ha permitido establecer distintos patrones de actuación y categorizar los diversos tipos de actividades que se realizan habitualmente en los centros educativos. Los resultados demuestran que el grueso de este tipo de experiencias no aprovechan todo el potencial educativo que realmente tiene la arqueología experimental. La amplia mayoría de experiencias analizadas no desarrollan actividades previas, de consolidación o de evaluación de la actividad. También se detecta que muchas se limitan a trabajar la prehistoria y, por tanto, se vinculan únicamente a los contenidos de $1 .^{\circ}$ ESO (12-13 años). Se propone finalmente un modelo estándar de actuación (PAEduca) de cara a que futuras actividades de este tipo puedan alcanzar un valor educativo más significativo y valioso, tanto para estudiantes como para docentes y centros educativos.

Abstract. During the last decades experimental archaeology workshops and experiences have multiplied their presence in Spanish schools and high schools. But, do they take advantage of their full educational potential? To answer this question a mixed methods study was designed. Different experimental archaeology projects that have been implemented in recent years in Spanish Secondary Education classrooms were analyzed. A non-probabilistic and nonrandom sampling was used as the study is based on the cases obtained through literature search, information found on the Internet and interviews with specialized companies. It was possible to establish different patterns of action and categorize the various types of activities that were usually carried out in educational centers. The results showed that most of these types of experiences did not take advantage of the full educational potential that experimental archaeology has proved to have. The vast majority of experiences analyzed did not introduce, consolidate or assess the experience by means of specific activities. It was also detected that many are limited to working on Prehistory and, therefore, were linked only to the contents of 1 st ESO (12-13 year olds). As conclusion, a standard model of action is proposed so that future activities of this type enable students to achieve a more significant and valuable educational outcomes.

\section{Palabras clave $\cdot$ Keywords}

Enseñanza secundaria, historia, ciencias sociales, investigación educativa, actividades escolares.

Secondary education, History, social sciences, educational Research, school activities. 


\section{Introducción}

La arqueología experimental es la disciplina encargada de estudiar los procesos de comportamiento de los restos materiales del pasado, a través de una reconstrucción experimental, de manera que se puedan extraer hipótesis para su posterior contraste con los datos arqueológicos. Los inicios de esta disciplina se vinculan claramente a la investigación y divulgación científica, pero sus cualidades pedagógicas y el uso de una metodología hipotética-deductiva, ha hecho de esta disciplina una herramienta muy válida también para un uso con fines meramente educativos (Ramos, 2007). Es por ello que, recientemente, ya son muchos los arqueólogos, empresas o docentes que han querido llevar la arqueología experimental a las aulas. Sin embargo, un estudio, sistematización y análisis de estas prácticas de arqueología experimental desde un punto de vista educativo aún estaba por hacer. ¿Qué se enseña? ¿Cómo se enseña? ¿Qué posibilidades se aprovechan? ¿Cuáles no?

\section{Marco teórico}

\subsection{La arqueología pública}

La arqueología experimental y su llegada a los centros educativos hay que contemplarla, sin duda, como fruto del crecimiento de la denominada arqueología pública. Este término, acuñado en la década de los 70 del siglo XX tras la publicación en EE. UU. del libro Public Archeology (McGimsey, 1972), sirvió para demostrar la importancia de conseguir la integración de las comunidades locales con el patrimonio, así como buscar reforzar la identidad y los vínculos con el pasado de dichos colectivos (Merriman, 2004). Desde un ámbito más europeo se redefinió esta idea y se empezó a hablar de una arqueología pública como canal de estudio de las relaciones entre arqueología y sociedad desde diversos ámbitos (Almansa, 2010) o, en otras palabras, cualquier actividad arqueológica que interactúa o puede interactuar con el público (Schadla-Hall, 1999). En particular, en España, la arqueología pública se ha cristalizado, sobre todo, en actividades de divulgación diversas como libros accesibles a todos los públicos, simulaciones arqueológicas o talleres variados. Sin embargo, estas aproximaciones se han realizado casi siempre desde un punto de vista meramente arqueológico.

Pero fusionar arqueología y educación demanda de un nuevo marco donde la óptica del especialista, del arqueólogo, no es suficiente, muy en el hilo de lo que se ha estudiado ya en relación al patrimonio o a la historia en general. La arqueología, como elemento patrimonial, encaja perfectamente en lo que serían las bases de la didáctica del patrimonio. Según estas es imprescindible procurar que los estudiantes comprendan las sociedades pasadas y presentes a través de esos elementos patrimoniales, desarrollar su propia habilidad interpretativa, influyendo así en su capacidad de valoración y, por tanto, en la conservación de los bienes (Cuenca, 2002; 2014). En este sentido, los programas de educación patrimonial cuentan, incluso, con su propio plan teórico y metodológico reafirmado e implementado en numerosos casos con estudios empíricos que han aportado resultados, lo cual ha permitido construir propuestas y estrategias diversas tanto en educación formal como no formal (Fontal y Ibáñez, 2015).

Ya son varios los trabajos que han defendido el uso de la arqueología experimental por favorecer un aprendizaje activo, dinámico o significativo (Masriera, 2008; Ramos, 2007; Villanueva et al. 2016). Sin embargo, a pesar de que el marco de actuación y la línea de investigación estén bien definidos, muchos de los proyectos de arqueología experimental, de arqueología en general, todavía no se vinculan a unos objetivos educativos claros. La razón quizás sea que los agentes relacionados con la difusión del patrimonio suelen recibir una formación más disciplinar (historia, arqueología), con escasa o ninguna carga educativa, salvo excepciones. A modo de ejemplo, el temario que habilita a los guías turísticos de la Región de Murcia cuenta con 28 temas, ninguno de ellos de corte didáctico (BORM, 24 de noviembre de 2017). Este hecho trasmite unos valores de conservación que carecen de elementos didácticos, imprescindibles para la correcta asimilación (Polo et al. 2014). Siguiendo con esta línea, el problema fundamental sería aún la clara división entre arqueólogos y educadores. Mientras que los arqueólogos se han centrado en mostrar y enseñar técnicas propias de la disciplina arqueológica sin tener en cuenta aspectos pedagógicos, los educadores, por el contrario, han buscado estimular el desarrollo cognitivo. Sería imprescindible tener en cuenta ambas perspectivas (González-Marcén, 2011). 


\subsection{Las posibilidades educativas de la Arqueología}

En España, tras la dictadura franquista, proliferaron grupos de innovación al amparo de los aires de cambio que llegaban de Reino Unido, destacando el grupo Historia 13-16. A este colectivo se debe la introducción de la simulación arqueológica, llegando a territorio peninsular en la década de los 80 del siglo XX (Santacana y Hernández, 1999). Al margen de la recreación arqueológica, surgen otra serie de propuestas que incorporan características o procesos propios de carácter arqueológico sin recreación de yacimientos arqueológicos en el centro o fuera del mismo (Egea y Arias, 2019). Esta visión de la arqueología con fines didácticos se vincula, sobre todo, en un principio con la denominada educación no formal, permitiendo desarrollar actividades y estrategias con mayor libertad al no estar estrechamente vinculado a un currículo predeterminado, siendo el alumnado el verdadero protagonista del proceso enseñanza-aprendizaje (Ibáñez et al., 2012).

Las posibilidades y estrategias educativas que posee la disciplina arqueológica son muchas y de muy diversa índole. La arqueología enseña los cambios que han sufrido las culturas humanas y su adaptación al territorio, factor este último que ha contribuido a la supervivencia como especie. La proliferación y puesta en común de la disciplina arqueológica en las aulas se argumenta por dos motivos fundamentales: por su carácter motivacional y por su carácter transversal, vinculándose con otras disciplinas (Henson, 2004; Santacana, 2018). Este componente interdisciplinar, combinado con el carácter procedimental (Zabala y Arnau, 2014), permite el trabajo de las competencias clave (Bardavio et al. 1996). A este carácter transversal se le suma claramente su potencial de atracción (Santacana et al. 2017). La metodología arqueológica favorece además la formación de ciudadanos críticos y mejor preparados para entender el presente (Egea y Arias, 2019), así como ciertas temáticas facilitan la comprensión de la naturaleza plural y diversa del pasado (Jiménez, 2017). En relación a cómo llevar la arqueología al aula, los modos de actuación disponibles son variados: excavaciones en yacimientos reales, simulados, museos de clase, kit-móviles, talleres, actividades en aula, proyectos, investigación-acción, didáctica con objetos, juegos de mesa, actividades virtuales, entre otras (Coma, 2011). Muchas de estas actividades llegan a través de diferentes agentes, tanto por el personal docente/departamento como arqueólogos reciclados o reconvertidos en docentes (González-Marcén, 2011). Más recientemente se han defendido incluso las posibilidades que la arqueología abre para el desarrollo de las denominadas habilidades del pensamiento histórico (Egea y Arias, 2018a; Wearing, 2011). Este último aspecto es clave pues recientes investigaciones demuestran el déficit que, en este sentido, poseen los estudiantes (Sáiz y Fuster, 2014).

\subsection{La arqueología experimental}

Dentro de la arqueología, la experimental hay que entenderla como parte del paradigma de la New Archeology, donde se puede considerar a Lewis R. Binford como uno de los padres de dicho modelo. Binford sostenía la idea de que la tarea arqueológica supone estudiar el proceso de la cultura (cómo, cuándo y por qué cambia la cultura humana) (Santacana y Hernández, 1999). Esta nueva visión de la arqueología se centraba en el análisis de cómo se construye el yacimiento arqueológico o cómo funcionan determinados útiles o herramientas (Ballart, 1997). Según Morgado y Baena (2011) la variabilidad de la experimentación arqueológica se puede resumir en dos grandes grupos. Por un lado, estaría todo lo relativo a la formación del registro arqueológico y, por el otro, todos esos experimentos relacionados con la realidad sociocultural del tiempo pasado. En este segundo grupo, entrarían las actividades sobre las materias primas con las que se elaboran las herramientas, sobre los procesos técnicos que se emplean, así como todo lo relativo a las formas de vida.

La práctica de la arqueología experimental, a diferencia de lo que ocurre en España, cuenta con una dilatada trayectoria en muchos países europeos, donde la investigación arqueológica confluye con la explotación turística, la puesta en valor de los bienes patrimoniales y sus posibilidades didácticas, creando modelos de simulación, tanto de formas de vida del pasado como de los procesos técnicos asociados a cada etapa (Masriera y Palomo, 2009). Esta experimentación activa permite aproximarse al conocimiento económico, social, tecnológico, cultural, ambiental y paisajístico de estas primeras comunidades (González et al. 1998). En España, la aplicación de la arqueología experimental con fines didácticos es un campo novedoso (desde finales del siglo XX), siendo en la actualidad un recurso muy utilizado en parques arqueológicos y museos (Alonso et al. 2010), ya sea mediante actividades manipulativas como talleres de fabricación de útiles, simulaciones de proyectos de investigación, etc. (González-Marcén, 2011). A pesar de ello, no es una disciplina exenta de problemas, ya que las actividades deben ser llamativas y permitir desarrollar múltiples habilidades como la observación, clasificación, pensamiento hipotético deductivo e incluso el análisis crítico (Cooper, 2013; Santacana, 2018). Por ello, si se enfoca la arqueología experimental 
desde un punto de vista didáctico, los objetivos deberían ser, entre otros: experimentar, aprender técnicas, incrementar la motivación, favorecer la reflexión, fomentar la cooperación entre iguales o fomentar valores de respeto al patrimonio (Bardavio, 1998).

Esta batería de aspectos, genéricos y específicos, son los que asegurarían el éxito de los talleres didácticos de arqueología experimental como oferta pedagógica, configurando un fenómeno en vías de desarrollo en España y con enorme importancia e influencia en países como Reino Unido o Francia. ¿Qué se puede hacer entonces? La arqueología experimental, entendida como herramienta auxiliar que recrea diferentes procesos de trabajo y manufactura, puede ser implementada describiendo la cultura material de ciertas culturas con dibujos o fotografías, analizar los objetos de manera descriptiva, elaborar hipótesis previas y posteriores a la actividad, intentar deducir funcionalidad y cronología, etc. (Bardavio, 1998).

Es importante destacar que la investigación a través de la experimentación se basa en la simulación, es decir, la realización de una serie de actividades orientadas a la comprobación u observación de procesos antrópicos o recreados sobre materiales que simulen situaciones, sucesos o procesos de estas sociedades pasadas. Tampoco es infrecuente encontrar bajo la etiqueta de "arqueología experimental" actividades más próximas a la teatralización y a la banalización (Masriera y Palomo, 2009). Pero la verdadera arqueología experimental supone la observación, análisis, reflexión e interpretación para su reproducción (Millán, 1997). Y en esta línea, el ámbito que está mostrando mayores propuestas desde el punto de vista didáctico es el de los procesos de elaboración de objetos y de obtención de productos. Como se podrá comprobar, desde este campo se trabajan actividades relacionadas con la tecnología prehistórica y protohistórica de forma casi exclusiva. Pero cabe recordar que también son posibles actividades experimentales de otras cronologías, como la romana, medieval, moderna o contemporánea, que, aunque su uso como recurso didáctico suele ser mucho menor (Ramos, 2007), permitirían potenciar el trabajo en valores, promoviendo el respeto al patrimonio y a la cultura, como, por ejemplo, acercando al alumnado a conocer los efectos de la guerra civil a través de las experiencias arqueológicas.

Pero desde un punto de vista educativo la práctica de la arqueología experimental, como se ha visto con las posibilidades educativas de la arqueología, puede ir más allá. Se puede lograr el aprendizaje conceptual (aquel relacionado con los aspectos históricos y arqueológicos de la actividad) pero también trabajar desde un punto de vista competencial, interdisciplinar, enseñar en valores (respeto al patrimonio, trabajo en equipo, etc.) y, por último, desarrollar las habilidades del pensamiento histórico (Egea y Arias, 2018b). Es en este punto en el que surge la pregunta de investigación de este trabajo: ¿Hasta qué punto se están aprovechando las posibilidades educativas de la arqueología en las actividades que se realizan en España de arqueología experimental?

Para responder a ello esta investigación planteó un objetivo general (OG) que consistió en: Diseñar un patrón de actuación para que los centros educativos, las empresas de arqueología o expertos afines puedan emplear a la hora de diseñar proyectos de arqueología experimental desde un punto de vista educativo. Pero para poder responder a este objetivo final, fue necesario responder previamente a los siguientes objetivos específicos (OE): (1) Identificar proyectos de arqueología experimental en centros de Educación Secundaria en España y (2) Categorizar y analizar dichos proyectos de arqueología experimental desde un punto de vista educativo.

\section{Método}

Se realizó una investigación de corte mixto con el fin de planificar y mejorar experiencias similares en un futuro (McMillan y Schumacher, 2005). La obtención de los proyectos a analizar se basó en un muestreo no probabilístico y no aleatorio en relación a la facilidad de acceso. Es decir, la búsqueda en repositorios digitales y en sitios web (de centros educativos y empresas) fue la que proporcionó finalmente el número de individuos (proyectos) a analizar. Para dicha búsqueda se emplearon términos de búsqueda como: "Arqueología Experimental", "talleres de Arqueología en centros de Secundaria", "talleres didácticos", "Arqueología en la Educación Secundaria", entre otros. En el caso de las empresas se pudo contactar personalmente con algunos de sus responsables, entrevistando a estos en base a los ítems de clasificación marcados por esta investigación, formulando una serie de preguntas tales como, ¿dónde suelen desarrollar sus actividades? ¿realizan actividades previas? ¿evalúan estas actividades?, entre otras cuestiones. El análisis se limitó a los centros de Secundaria de cara a que los proyectos a comparar fueran más similares y, por lo tanto, el análisis por categorías fuera más consistente. La información se recogió en una base de datos (Microsoft Access), diferenciando entre (1) proyectos de arqueología experimental (PAE) desarrollados en los centros de Educación Secundaria y (2) patrones de clasificación, para un posterior análisis y descripción de cada uno de los centros de Educación Secundaria. 
En el caso de la base de datos destinada a sistematizar los PAE, se diferenció entre los siguientes campos: Código (los nombres de los centros se anonimizaron), año de realización, ubicación (provincia), cronología que se trabaja y curso en el que se desarrolló la actividad (Tabla 1).

Tabla 1

Parrilla de Identificación de Proyectos de Arqueología Experimental

\begin{tabular}{ll}
\hline Categoría & Subcategorías \\
\hline Código & PAE XX \\
\hline Año & Campo abierto. \\
\hline Cronología & Prehistoria (Paleolítico, Neolítico, Edad de los Metales) \\
\hline Curso & Historia (Historia Antigua, Historia Medieval, etc.) \\
\hline & $1 .^{\circ}-4 .^{\circ}$ ESO; $1 .^{\circ}-2 .^{\circ}$ Bachillerato.
\end{tabular}

Nota: PAE (Proyecto de Arqueología Experimental).

Por lo que respecta a la parrilla relativa al análisis de los proyectos, desde un punto de vista educativo, la información recogida fue codificada empleando un sistema nominal de categorías combinado con algunos campos abiertos. Se diferenciaron las siguientes categorías y subcategorías (Tabla 2):

Tabla 2

Parrilla de Identificación de Proyectos de Arqueología Experimental desde un punto de vista educativo

\begin{tabular}{ll}
\hline Categoría & Subcategorías \\
\hline Espacio & Aula / Patio / Exterior \\
\hline Actividades Previas & Sí / No \\
\hline Tipos de Actividades Previas & $\begin{array}{l}\text { Método expositivo / Método interrogativo / Estrategias de indagación } \\
\text { / Trabajo de investigación en grupo / Trabajos individuales / Técnicas } \\
\text { cooperativas }\end{array}$ \\
\hline Descripción de la actividad & Campo abierto \\
\hline Actividades de consolidación & Sí / No \\
\hline Personal responsable & $\begin{array}{l}\text { Actividades realizadas por el propio docente/departamento / } \\
\text { Actividades realizadas por un arqueólogo profesional / Empresa } \\
\text { externa }\end{array}$ \\
\hline
\end{tabular}

Evaluación de la actividad Sí / No

Tipos de evaluación Campo abierto si procede.

Tipos de aprendizaje Aprendizaje conceptual / Aprendizaje interdisciplinar / Aprendizaje competencial / Aprendizaje en valores / Trabajo del pensamiento histórico

La unidad principal de análisis fueron los proyectos relacionados con la introducción de la arqueología experimental en centros de Educación Secundaria (PAE). Se trata de una investigación de carácter cualitativo a partir de la aplicación de los patrones de clasificación (categorías) antes mencionados a cada uno de los proyectos localizados. Cabe destacar la categorización realizada sobre los tipos de aprendizaje que ofrecía cada uno de los proyectos (Tabla 3). La elección viene justificada ante el evidente vacío bibliográfico que presentan los planteamientos y prácticas de talleres experimentales en las aulas. A pesar de que la información localizada ha sido escueta, se han logrado analizar once casos repartidos por diferentes provincias españolas.

Tabla 3

Tipos de aprendizaje y ejemplos de categorización de los proyectos analizados

\begin{tabular}{lll}
\hline Tipos de & Definición & Ejemplos
\end{tabular}




\section{aprendizaje}

Aprendizaje conceptual

Aprendizaje interdisciplinar
Aquel relacionado con los aspectos $\mathrm{Si}$ predominan tareas relativas a la históricos y arqueológicos de la actividad. terminología arqueológica o a hechos concretos.

\section{Aquel relacionado con la Si incorporan aspectos de otras interdisciplinariedad de los conocimientos. disciplinas tales como la Geología (industria lítica) o la antropología (material óseo).}

Aprendizaje competencial
Aquel relacionado con las competencias básicas del Currículo.
Si las estrategias permiten el desarrollo de competencias básicas como la comunicación lingüística, aprender a aprender, etc.
Aprendizaje en valores
Aquel relacionado con la transmisión de los Si se hace hincapié en la importancia de valores de respeto al patrimonio y a la respetar y valorar los bienes cultura, trabajo en equipo, etc. patrimoniales o hay cierto peso en tareas en las que se fomente la cooperación y trabajo en equipo.

Trabajo del pensamiento histórico
Es aquel relacionado con la reflexión de las Si se establecen tareas que fomenten la habilidades que el aprendizaje de la Historia perspectiva, la relevancia, el método de y la Arqueología aporta al alumnado. trabajo con las fuentes, procesos de causa y consecuencia, etc.

\section{Resultados y discusión de resultados}

Objetivo 1: Identificar proyectos de arqueología experimental en centros de Educación Secundaria en España. La sistematización de los PAE aportó como resultado la siguiente tabla (Tabla 4).

Tabla 4

Listado de Proyectos de Arqueología Experimental analizados

\begin{tabular}{lllll}
\hline Código & Año & Ubicación & Cronología & Curso \\
\hline PAE01 & 1996 & Barcelona & Neolítico & No se indica \\
\hline PAE02 & 2014 & Madrid & Neolítico & PCPI \\
\hline PAE03 & $2015 ; 2018$ & Castellón & Paleolítico & $1 .^{\circ}$ ESO \\
\hline PAE04 & 2015 & Pontevedra & Edad de los Metales & Diversos cursos \\
\hline PAE05 & 2018 & Las Palmas & Neolítico & Diversos cursos \\
\hline PAE06 & 2018 & Madrid & Paleolítico & $1 .^{\circ}$ ESO \\
\hline PAE07 & 2019 & Murcia & Paleolítico; Neolítico & $1 .^{\circ}$ ESO \\
\hline PAE08 & 2019 & Zaragoza & Paleolítico & $1 .^{\circ}$ ESO \\
\hline PAE09 & 2019 & Valladolid & Calcolítico; Edad del Bronce & $1 .^{\circ}$ ESO \\
\hline PAE10 & - & Zaragoza & Edad de los Metales & $1 .^{\circ}$ ESO \\
\hline PAE11 & - & Barcelona & Paleolítico & $1 .^{\circ}$ ESO \\
\hline Not: PAE & & & \\
\hline
\end{tabular}

Nota: PAE (Proyecto de Arqueología Experimental). 
Atendiendo a la fecha de realización de estos proyectos, se ha detectado una característica común a todos ellos, el diseño y la implementación de estas actividades se han llevado a cabo durante la última década, exceptuando el PAE01 (1996). Este aspecto responde a la progresiva entrada de las actividades de carácter arqueológico en las aulas de secundaria españolas (Egea y Arias, 2018a).

Geográficamente se observa cierta heterogeneidad en cuanto a la ubicación de los PAE de la muestra, distribuidos por toda la geografía española: Cataluña, Comunidad de Madrid, Castilla-La Mancha, Castilla y León, Aragón, Región de Murcia, Galicia e Islas Canarias.

Es digno de mención que en todos los PAE han trabajado contenidos prehistóricos -desde el Paleolítico a la Edad de los Metales-, convirtiéndose en una herramienta que facilita la comprensión del conjunto de actividades desarrolladas por el ser humano a lo largo de la prehistoria. En esta línea, se reafirma lo apuntado por Prats y Santacana (2011), limitando la presencia de la arqueología a etapas prehistóricas o antiguas. Dicha exclusividad en estos periodos se entiende por la escasa tradición de un cuerpo de historiadores que no tiende a profundizar en aspectos materiales y por la existencia de otras alternativas como las fuentes documentales para la aplicación didáctica (Bardavio et al. 2001).

En la mayor parte de los PAE analizados se han abordado contenidos de cronología paleolítica y neolítica, representando entre ambas etapas casi la totalidad de la muestra. Entre los tipos de actividades realizadas, destacamos los talleres de tecnología prehistórica, presentes en la gran parte de los casos $(\mathrm{n}=6$, $54,5 \%$ ), donde se ha procedido a la identificación de diferentes útiles y a la realización de prácticas sencillas como la talla de núcleos con percutores de sílex o cuarcita, fabricando algunas piezas.

Por otro lado, los PAE vinculados a Edad de los Metales son más minoritarios (PAE04 y PAE10), realizando actividades de elaboración de alimentos (con horno y molinos) y de tipo manufacturero (fundiendo cobre dentro de un pequeño recipiente cerámico) respectivamente. Por último, se registra un proyecto vinculado al Calcolítico y Edad del Bronce, concretamente al dibujo de material cerámico (PAE09).

Estos proyectos, por regla general, están asociados a $10^{\circ}$ de ESO, curso donde se imparten los contenidos curriculares de carácter prehistórico, como así se evidencia en la mayoría de casos ( $\mathrm{n}=7,63,6 \%$ ), exceptuando ciertos centros donde estos proyectos iban dirigidos para otros cursos (como en el caso del PAE02, destinado para el alumnado de PCPI del centro) o la congregación de varios cursos académicos para asistir a estas actividades (PAE04 y PAE05). Por último, en PAE01 no se especifica la información del curso al que va dirigido.

Objetivo 2: Categorizar los proyectos de arqueología experimental desde un punto de vista educativo. Se procede ahora a resumir los principales datos relativos a cómo se desarrollan este tipo de actividades desde un punto de vista educativo. La parrilla general se resume mediante la siguiente tabla (Tabla 5).

Tabla 5

Resumen de la parrilla de categorización de los PAE

\begin{tabular}{|c|c|c|c|c|c|}
\hline Código & $\begin{array}{l}\text { Act. } \\
\text { Previas }\end{array}$ & $\begin{array}{l}\text { Tipo } \\
\text { Act.Prev }\end{array}$ & $\begin{array}{l}\text { Act. } \\
\text { Consolid. }\end{array}$ & Evaluación & $\begin{array}{l}\text { Tipo } \\
\text { Aprendizaje }\end{array}$ \\
\hline$\overline{\text { PAE01 }}$ & Sí & Técnicas cooperativas & No & Sí & $\begin{array}{l}\text { A.CON. } \\
\text { A.COMP. } \\
\text { A.INT. }\end{array}$ \\
\hline PAE02 & Sí & Método expositivo & No & No se menciona & $\begin{array}{l}\text { A.CON. } \\
\text { A.VAL. } \\
\text { A.INT. }\end{array}$ \\
\hline PAE03 & No & - & No & No se menciona & $\begin{array}{l}\text { A.CON. } \\
\text { A.COMP. } \\
\text { A.VAL. } \\
\text { A.INT. }\end{array}$ \\
\hline PAE04 & Sí & Método expositivo & Sí & No se menciona & $\begin{array}{l}\text { A.CON. } \\
\text { A.VAL. } \\
\text { A.INT. }\end{array}$ \\
\hline$\overline{\mathrm{PAE} 05}$ & No & - & No & No se menciona & $\begin{array}{l}\text { A.VAL. } \\
\text { A.INT. }\end{array}$ \\
\hline$\overline{\text { PAE06 }}$ & No & - & No & No se menciona & $\begin{array}{l}\text { A.COMP. } \\
\text { A.CON. } \\
\text { A.VAL. } \\
\text { A.INT. }\end{array}$ \\
\hline
\end{tabular}




\begin{tabular}{|c|c|c|c|c|c|}
\hline & & & \multicolumn{2}{|c|}{ Investigación en la Escuela, no 103, 202} & 16 \\
\hline$\overline{\mathrm{PAE} 07}$ & Sí & Método expositivo & No & No se menciona & $\begin{array}{l}\text { A.COMP. } \\
\text { A.CON. } \\
\text { A.VAL. } \\
\text { A.INT. }\end{array}$ \\
\hline$\overline{\text { PAE08 }}$ & Sí & Método expositivo & No & No se menciona & $\begin{array}{l}\text { A.COMP. } \\
\text { A.CON. } \\
\text { A.VAL. } \\
\text { A.INT. }\end{array}$ \\
\hline$\overline{\text { PAE09 }}$ & Sí & $\begin{array}{l}\text { Método expositivo e } \\
\text { interrogativo }\end{array}$ & No & No se menciona & $\begin{array}{l}\text { A.COMP. } \\
\text { A.CON. } \\
\text { A.VAL. }\end{array}$ \\
\hline$\overline{\text { PAE10 }}$ & Sí & Método expositivo & No & No se menciona & $\begin{array}{l}\text { A.COMP. } \\
\text { A.CON. } \\
\text { A.VAL. } \\
\text { A.INT. }\end{array}$ \\
\hline$\overline{\text { PAE11 }}$ & Sí & $\begin{array}{l}\text { Método expositivo e } \\
\text { interrogativo }\end{array}$ & No & No se menciona & $\begin{array}{l}\text { A.COMP. } \\
\text { A.CON. } \\
\text { A.VAL. }\end{array}$ \\
\hline
\end{tabular}

Nota: El sistema de abreviaturas utilizado para el tipo de aprendizaje es el siguiente. PAE (Proyecto de Arqueología Experimental). A.CON. (Aprendizaje conceptual); A. INT. (Aprendizaje interdisciplinar; A.COMP. (Aprendizaje competencial); A.VAL. (Aprendizaje en valores); P.H. (Trabajo del pensamiento histórico).

En relación al espacio, el aula habitual de clase o algún espacio interior del centro han sido los lugares elegidos en la mayoría de centros para desempeñar los PAE ( $\mathrm{n}=9,81,8 \%$ ). Por el contrario, en otros dos PAE el espacio utilizado ha sido el patio del centro -PAE03 y PAE10- ( $\mathrm{n}=2: 18,2 \%)$. Con respecto al personal encargado del diseño y de la implementación de estas actividades, hay cierta heterogeneidad entre las realizadas por el propio docente/departamento, por un arqueólogo profesional externo y por empresas externas dedicadas a este tipo de actividades, tal como apuntaba González-Marcén (2011), aunque con un cierto predominio de estas últimas.

El desarrollo de las actividades de los PAE se divide en: previas, realizadas y de consolidación. En cuanto a las actividades previas, destaca la presencia de estas en la mayoría de PAE ( $\mathrm{n}=8,73 \%$ ). Por regla general se han desarrollado a través de una metodología expositiva de enseñanza, (PAE02, PAE04, PAE0711), combinando esta, en ocasiones, con una metodología interrogativa para captar la atención e interés del alumnado. Esta metodología expositiva de tipo teórico se ha focalizado en la introducción al alumnado a la disciplina arqueológica, recorrido por etapas prehistóricas y, en concreto, a las posibilidades que ofrece la arqueología experimental. La excepción se encuentra en el PAE01 donde las actividades previas han seguido una estrategia diferente, a través de un trabajo de investigación en grupo y técnicas cooperativas con la realización de una excavación simulada.

La parte central de las actividades de arqueología experimental siguen un patrón bastante común: una demostración por parte del personal encargado de la ejecución de la actividad y una posterior experimentación del alumnado con los materiales proporcionados. A través de esta metodología, el alumnado comprende el proceso de elaboración de los útiles y las posibilidades que ofrecen desde un punto de vista práctico. Este tipo de estrategias de indagación permiten al alumnado desarrollar acciones propias del proceso enseñanza-aprendizaje siguiendo las pautas y técnicas proporcionadas. Estos proyectos siguen la línea que apunta Coma (2011).

Pero en contraposición con la existencia de actividades previas, las actividades de consolidación están presentes en un único PAE ( $\mathrm{n}=1,9,1 \%$ ). Esta ausencia se denota en que las actividades están programadas para un día concreto y para una hora determinada, es decir, no se profundiza mucho más en el contenido expuesto por falta de tiempo y, por tanto, es muy complicado consolidar el aprendizaje (Diaz-Barriga y Hernández, 2010).

Todos los PAE presentan una característica común, la no evaluación de las actividades desarrolladas en sus proyectos, a excepción del PAE01, que emplea fichas de catalogación de material. Esta ausencia de evaluación puede responder al carácter práctico y actitudinal de los proyectos, enfocados sobre todo en la transmisión de los valores del respeto al patrimonio, trabajo en equipo y fomento de actitudes y actividades de colaboración y participación. Por lo tanto, poco o nada se puede decir de los tipos de evaluación, uno de los pilares fundamentales de cualquier tipo de estrategia educativa (Sanmartí, 2010).

Respecto al tipo de aprendizaje, se detecta la presencia de los cuatro tipos-competencial, conceptual, en valores e interdisciplinar- en la mayor parte de los PAE de la muestra. Son mayoritarios el aprendizaje 
conceptual $(n=10,91 \%)$ y el aprendizaje en valores $(n=10,91 \%)$. El primero de ellos, presente en casi todos los PAE de la muestra, dota al alumnado de una terminología arqueológica e histórica con la que apoyarse en el transcurso de los talleres. El segundo es un activo muy importante en este tipo de actividades, relacionadas con la valoración y respeto del patrimonio histórico-arqueológico (Estepa et al. 1998). Estas actividades tienen un fuerte componente actitudinal, es decir, están relacionadas con valores identitarios como el respeto, convivir en comunidad, trabajo en equipo, etc. (Quijano-Araníbar, 2018). No obstante, a pesar de que el aprendizaje competencial, el conceptual y en valores tengan la misma frecuencia, la carga del aprendizaje actitudinal o en valores es mayor que la del resto de aprendizajes. Otro de los rasgos que caracteriza a estos PAE es la interdisciplinariedad de sus contenidos ( $\mathrm{n}=9,81,2 \%$ ), ya que se incorporan otros aspectos y competencias propias de otras disciplinas como las Ciencias Sociales, Ciencias Naturales, Tecnología, etc. (Santacana, 2018). Seguidamente estaría el aprendizaje de tipo competencial ( $\mathrm{n}=8,73 \%$ ). La enseñanza de la arqueología cuenta con ciertas características que hacen de la misma una herramienta eficaz para la enseñanza por competencias (Egea y Arias, 2013; Zabala y Arnau, 2014), tales como la significatividad y el carácter procedimental de estas actividades. En último lugar, cabría subrayar que ningún PAE alude directamente o refleja indicios de intentar estar trabajando el pensamiento histórico.

Por lo tanto, a modo de síntesis, se puede decir que la gran mayoría de PAE ( $\mathrm{n}=6,55 \%$ ) -PAE02; PAE07-PAE11- cumplen todos los patrones de clasificación establecidos. Sí que es cierto que faltan dos elementos tan fundamentales como son las actividades de consolidación y la evaluación de la actividad. En otro nivel estaría el resto de PAE ( $\mathrm{n}=3$, 27 \%) -PAE03; PAE05; PAE06- donde no se cumplen los siguientes campos: actividades previas, actividades de consolidación, evaluación de la actividad.

Objetivo General: Diseñar un patrón de actuación para los PAE en centros de Secundaria. Una vez analizadas y discutidas las particularidades de cada PAE de la muestra en base a los patrones de clasificación establecidos, se concluye proponiendo el modelo PAEduca (Proyectos de Arqueología Experimental Educativos). Este modo de trabajo establece las bases de actuación para llevar a cabo el proceso de enseñanza-aprendizaje de la arqueología experimental en las aulas, respondiendo así al objetivo principal marcado por esta investigación (Figura 1). 
Figura 1

Infografía que resume los principios claves para organizar una experiencia de arqueología experimental desde un punto de vista educativo. Modelo PAEduca.

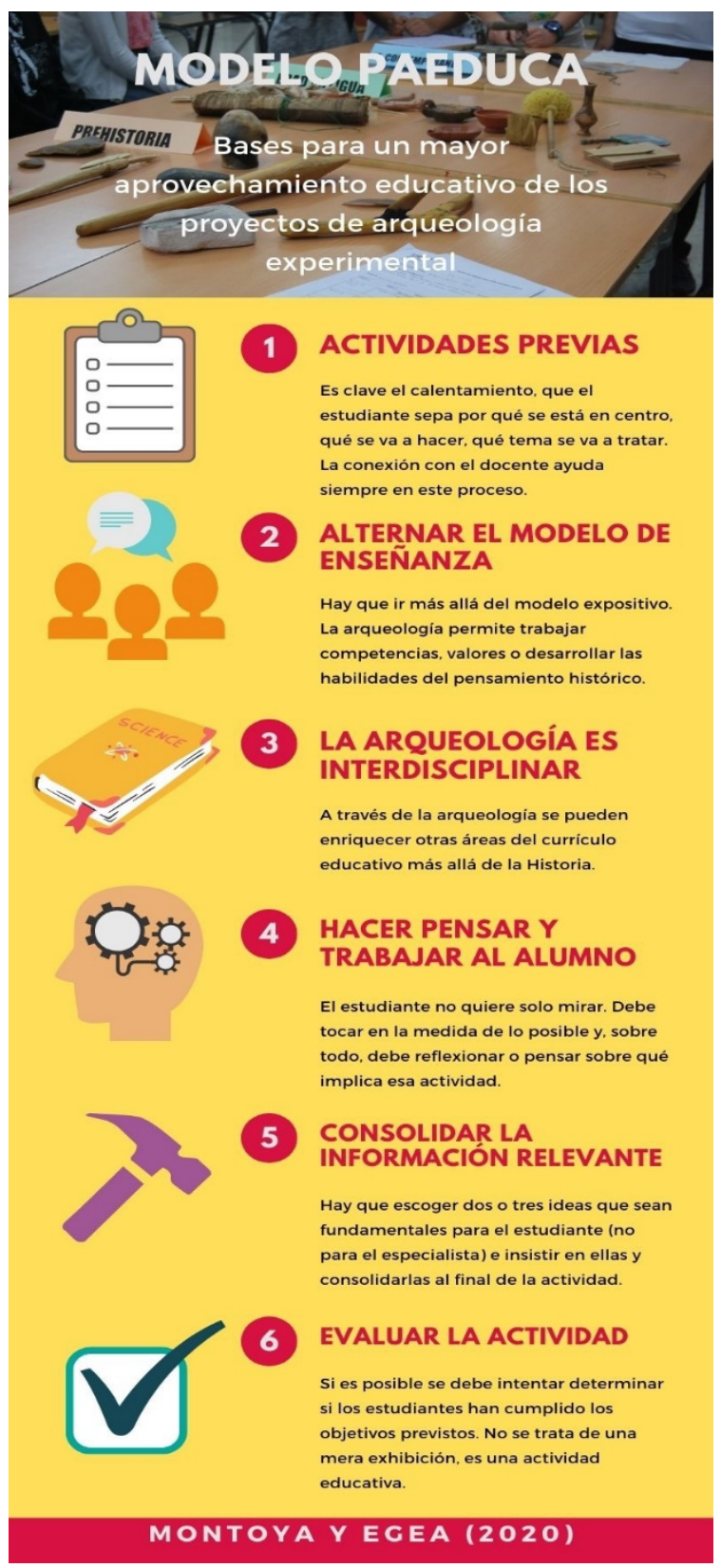

No es baladí que el espacio o lugar de realización de la actividad (aula, patio, exterior) debe estar acondicionado: amplias mesas propicias para el trabajo cooperativo, libertad de movimientos para los estudiantes, agua corriente disponible, lupas binoculares, etc., es decir, adecuar el espacio para convertirlo en un auténtico laboratorio de historia (arqueología). Otras materias de Secundaria disponen de este espacio propio mientras que Geografía e Historia no (Santacana, 2005).

Pero quizás el primer punto clave sea la realización de actividades previas al desarrollo de los PAE. Es clave contextualizar la actividad e impartir algunas nociones básicas sobre arqueología y el período que se va a trabajar. Para su puesta en valor se pueden emplear diversos métodos de enseñanza de forma alternativa o simultánea sin necesidad de limitarnos al método expositivo más habitual. Así será posible adaptar este tipo de proyectos a métodos interrogativos, estrategias de indagación, trabajos de investigación individuales o grupales o técnicas cooperativas.

Seguidamente, a la hora de plantear las actividades propias de estos proyectos -ya sean talleres de tecnología prehistórica, talleres de producción manufacturera, talleres de dibujo arqueológico- habrá que tener en cuenta la naturaleza de estas, eligiendo o elaborando aquellas que cumplan con el carácter transversal, interdisciplinar y didáctico de la arqueología experimental. El personal encargado de la 
realización de la actividad debería proceder a la identificación y delimitación de la realidad observada, situando espacial y temporalmente al objeto y/o útiles en cuestión. Tras ello, se procederá a la ejecución de la actividad diseñada previamente. Los PAE trabajarán los siguientes aspectos, de forma individual o simultánea: (1) experimentos sobre las propiedades y comportamientos físico-químicos de la materia prima de los útiles; (2) experimentación sobre la tecnología, procesos técnicos; (3) experimentación sobre los modos de vida (Morgado y Baena, 2011). Dependiendo de la naturaleza de cada una de las iniciativas se seguirán unas líneas de actuación u otras, pero, en líneas generales, la actividad deberá constar de dos partes: (1) explicación y demostración por parte del personal encargado de la actividad, (2) sin olvidar la experimentación por parte del alumnado, en la que ellos comprueban por sí mismos el proceso de elaboración/fabricación del útil (Bardavio, 1998). Si la arqueología experimental es "un modelo de contrastación de hipótesis a través de la experimentación que de forma rigurosa admita la validez" (Baena, 1997, p. 3), una actividad educativa de este tipo debe servir para que los estudiantes saquen hipótesis previas y posteriores a la observación y contrasten también las mismas. Deberían ser capaces de enumerar o describir de manera sencilla las relaciones existentes entre los diferentes aspectos observados, junto a una posterior interpretación personal. No basta con la mera explicación práctica o la exhibición. El alumnado puede describir los aspectos observados a través de dibujos, esquemas, fotografías, etc., trasladando sus conocimientos a la práctica a través de la experimentación y análisis del material.

El diseño de estos PAE puede orientarse hacia la consecución de diferentes tipos de aprendizaje que vayan más allá del contenido de tipo conceptual. De manera concreta con este tipo de PAE podrían lograrse diferentes tipos de aprendizaje (Tabla 6).

Tabla 6

Posibles tipos de aprendizaje a desarrollar con actividades de arqueología experimental

\begin{tabular}{ll}
\hline Tipo de aprendizaje & Modo de conseguirlo \\
\hline Aprendizaje conceptual & $\begin{array}{l}\text { Se podrían trabajar términos como fuentes primarias, arqueología, } \\
\text { arqueología experimental, materias primas, periodización histórica, } \\
\text { prehistoria, modos de vida, etc. }\end{array}$ \\
\hline Aprendizaje interdisciplinar & $\begin{array}{l}\text { Se consigue incorporando aspectos de otros campos o disciplinas } \\
\text { escolares, como, por ejemplo, el tratamiento del material lítico de la } \\
\text { Geología o el estudio del material óseo de la Antropología, entre otros. }\end{array}$ \\
\hline Aprendizaje competencial & $\begin{array}{l}\text { Son fácilmente desarrollables la comunicación lingǘstica, aprender a } \\
\text { aprender, competencias sociales y cívicas, sentido de iniciativa y espíritu } \\
\text { emprendedor, conciencia y expresiones culturales. }\end{array}$ \\
\hline Aprendizaje en valores & $\begin{array}{l}\text { Se trataría de fomentar la transmisión de los valores del respeto al } \\
\text { patrimonio, rechazo de prejuicios hacia otros modelos de sociedad, } \\
\text { trabajo en equipo y fomento de actitudes y actividades de colaboración y } \\
\text { participación. }\end{array}$ \\
\hline $\begin{array}{l}\text { Habilidades del pensamiento puede trabajar sin dificultad la relevancia histórica, usar pruebas } \\
\text { históricas, cambio y continuidad, perspectiva histórica, causa y } \\
\text { consecuencia dimensión ética (Egea y Arias 2018b). }\end{array}$ \\
\hline
\end{tabular}

Tras la ejecución del proyecto es clave desarrollar una serie de actividades que consoliden el conocimiento adquirido. Se deben analizar los resultados obtenidos para verificar las hipótesis establecidas al inicio de la actividad, buscando la participación activa del estudiante. Ya hay algunas propuestas interesantes al respecto (Villanueva et al. 2016). Sería muy útil reseñar en conjunto los aspectos más importantes de cada iniciativa o incluso afianzar los contenidos a través del empleo de material audiovisual o algún tipo de exposición fotográfica como en el caso del PAE04 -única iniciativa de la muestra donde se denota la presencia de actividades de consolidación-. El empleo de varios canales para hacer llegar el mensaje que se quiere transmitir repercute en una mejor retención (Seel, 2008).

Sería conveniente además proponer algún tipo de actividad de evaluación para comprobar el nivel atención y comprensión del alumnado en estas iniciativas. Esta evaluación puede hacerse durante el desarrollo del taller o puede coordinarse con el docente para que sea realizada más adelante. En el caso de los PAE, al tratarse de iniciativas eminentemente prácticas, se proponen las siguientes: observación de las actividades del alumnado mediante fichas individuales, pruebas orales, entrevistas y cuestionarios, actividades de autoevaluación y coevaluación, etc. 


\section{Conclusiones}

Pese haber podido responder a los objetivos marcados al inicio de esta investigación, no ha sido tarea fácil recopilar la información necesaria para ello. La dificultad ha residido en las pocas experiencias en relación a esta temática, factor que se une seguramente a los pocos ejemplos publicados o en abierto. Aun así, se ha comprobado cómo la arqueología experimental ha ido ganando protagonismo en algunos programas educativos con el paso del tiempo. La mayoría de ellos se realizan en los ámbitos de educación no formal pero esta investigación ha puesto el foco en aquellos proyectos del ámbito formal. Se considera que se trata de un estudio de relevancia ya que el análisis demuestra que, la mayoría de los proyectos analizados, cumplen casi todos los ítems marcados, lo cual es muy esperanzador.

Sin embargo, se aprecia que, en gran parte de las iniciativas, no se realizan actividades previas, de consolidación y evaluación de la actividad. Estas premisas, tal y como se defiende en el modelo de buenas prácticas que se ha diseñado (Figura 1), deberían considerarse para su puesta en práctica, ya que resulta fundamental dotar de unas nociones previas al alumnado, presentando la actividad a través del recorrido por la cultura o épocas que se vayan a trabajar (actividades previas). Por otro lado, la realización de actividades que consoliden el conocimiento adquirido sería óptimo para verificar las hipótesis establecidas al inicio de la actividad, afianzando el contenido impartido a través del empleo de material audiovisual. En cuanto a la evaluación de estos proyectos, sería interesante proponer algún tipo de actividad para comprobar el nivel de comprensión del alumnado, reflejando sus conocimientos a través de cualquier tipo de prueba como las que aquí se proponen, evaluando bajo el marco que proponga la legislación de cada autonomía. En cuanto a las limitaciones de la investigación cabe destacar la posibilidad de mejora de la muestra escogida. Así como se podría ampliar el número de ítems establecidos o incluso entrevistar al alumnado de los centros para conocer sus impresiones sobre la temática. Futuras investigaciones podrán corroborar o no las bases que aquí se han establecido. Pero se considera que el modelo de buenas prácticas de arqueología experimental que aquí se presenta puede ser útil para la puesta en valor de futuros proyectos o iniciativas de arqueología experimental.

\section{Referencias}

Almansa, J. (2010). Pre-editorial: Towards a Public Archaeology. AP: Online Journal in Public Archaeology, 0, 1 3.

Alonso, R., Terradillos, M. y Díez, J. C. (2010). Arqueología experimental, una herramienta para el conocimiento de la prehistoria. En J.A. Meneses, M.A. Moreira y C. Caballero (Eds.), III Encuentro internacional sobre investigación en enseñanza en ciencias (pp. 545-556). Universidad de Burgos.

Baena, J. (1997). Arqueología experimental, algo más que un juego. Boletín de Arqueología Experimental, 1, 25.

Ballart, J. (1997). El patrimonio bistórico y arqueológico: valor y uso. Ariel.

Bardavio, A. (1998). Arqueología experimental en la educación secundaria obligatoria. Revista de Arqueología, 208, 6-15.

Bardavio, A., Gatell, C. y Molinero, F. (1996). Recerca i divulgació arqueològica. Un camí per al desenvolupament d'estratègies interdisciplinars a l'ESO. Treballs d'Arqueologia, 4, 50-76.

Bardavio, A., González, P., González, J., y Masvidal, C. (2001). Arqueologia experimental i les seves aplicacions didàctiques: projectes entorn a l'arquitectura prehistòrica al Vallès (Barcelona). En M. Carme, J. Pou, J. Sanmartí, y J. Santacana (Eds.), Tècniques constructives d' època ibèrica $i$ experimentació arquitectònica a la Mediterrània: Actes de laI Reuniò Internacional d' Arqueologia de Calafell (Calafell, 20, 21 i 22 de gener del 2000) (pp.43-58). Universidad Autónoma de Barcelona.

BORM (24 de noviembre de 2017). Resolución de 7 de noviembre de 2017 del Instituto de Turismo de la Región de Murcia por la que se convocan pruebas para la obtención de la habilitación de guía de turismo de la Región de Murcia y en algún idioma extranjero para guías ya habilitados. Boletín Oficial de la Región de Murcia, 272, 32429-32437. Recuperado de https://bit.ly/2K5BXjR

Corbishley, M. (2011). Pinning down the past: archaeology, heritage, and education today. Woodbridge, Suffolk, UK; Rochester, NY: Boydell Press

Cooper, H. (2013). Why must teaching and learning in history be creative? In H.Cooper (Ed.), Teaching history creatively (pp. 3-18). Routledge.

Coma, L. (2011). Actividades educativas y didácticas del patrimonio en las ciudades españolas (Tesis Doctoral). Universidad de Barcelona. 
Cuenca, J. M. (2002). El patrimonio en la didáctica de las ciencias sociales: análisis de concepciones, dificultades y obstáculos para su integración en la enseñanza obligatoria (Tesis doctoral). Universidad de Huelva. Recuperado de https://bit.ly/3lpvsWD

Cuenca, J. M. (2014). El papel del patrimonio en los centros educativos: hacia la socialización patrimonial. Tejuelo, 19, 76-96.

Diaz-Barriga, F. y Hernández, G. (2010). Estrategias docentes para un aprendizaje significativo: Una interpretación constructivista. McGraw-Hill Interamericana.

Egea, A., y Arias, L. (2013). IES Arqueológico: La arqueología como recurso para trabajar las competencias básicas en la educación secundaria. Clio: History and History Teaching., 39.

Egea, A. y Arias, L. (2018a). El desafío de enseñar a pensar históricamente a través de la arqueología. En A. Egea, L. Arias y J. Santacana (Eds.), Y la arqueología llegó al aula. La cultura material y el método arqueológico para la enseñanza de la historiay el patrimonio (pp. 327-341). Trea.

Egea, A. y Arias, L. (2018b). Aprendizaje basado en objetos en Educación Secundaria: primeros resultados de una experiencia didáctica. En C. López, C. García Ruiz y M. Sánchez Agustí (Eds.), Buscando formas de enseñar; investigar para innovar en didáctica de las Ciencias Sociales (pp. 919-932). Universidad de Valladolid.

Egea, A. y Arias, L. (2019). Enseñanza y Arqueología ¿un diálogo posible? En L. Coma, y T. Martínez (Eds.), Joan Santacana Mestre, Al savi profesor, arqueóleg i museòleg (pp. 165-176). Barcelona.

Estepa, J., Domínguez, C. y Cuenca, J. M. (1998). La enseñanza de valores a través del patrimonio. En A. M. Filella Pujol (Ed.), Los valores y la didáctica de las Ciencias Sociales: Actas del IX Simposium de Didáctica de las Ciencias Sociales (pp. 327-336). AUPDCS. Recuperado de https://bit.ly/3iIO3ex

Fontal, O. y Ibáñez, A. (2015). Estrategias e instrumentos para la educación patrimonial en España. Educatio Siglo XXI, 3, 15-32.

González-Marcén, P. (2011). La dimensión educativa de la Arqueología. Congreso de Prebistoria de Andalucia (pp. 497-506). Junta de Andalucía.

González, P., Castañeda, N., Armentano, N., Barahona, M. y González, J. (1998). La recerca a l'abast: l'experiència del Parc Archeològic del Patronat Flor de Maig. Treballs d'Arqueologia, 5, 65-84.

Henson, D. (2004). Archaeology and education, an exercise in constructing the past. Treballs d'Arqueologia, $10,5-16$.

Ibáñez, A., Vicent, N. y Asensio, M. (2012). Aprendizaje informal, patrimonio y dispositivos móviles. Evaluación de una experiencia en educación secundaria. Didáctica de las Ciencias Experimentales y Sociales, 26, 3-18.

Jiménez, H. (2017). El patrimonio fenicio-púnico. Claves para su socialización, puesta en valor y uso didáctico. Panta Rei. Revista de Ciencia y Didáctica de la Historia, 85-107. https://doi.org/10.6018/pantarei/2017/6

Masriera, C. (2008). Presentación del patrimonio arqueológico: Ruinas "versus" reconstrucciones, ¿qué entiende más el público? Íber. Didáctica de las Ciencias Sociales, Geografía e Historia, 57, 39-51.

Masriera, C. y Palomo, A. (2009). Arqueología experimental i difusió. Cota zero, 24, 31-38.

McGimsey, C. R. (1972). Public archeology. Seminar Press.

McMillan, J. H. y Schumacher, S. (2005). Investigación educativa: Una introducción conceptual. Pearson.

Merriman, N. (Ed.). (2004). Public archaeology. Routledge.

Millán, M. D. (1997). La simulación y la representación de la realidad. En A. de la Torre (Eds.), Estrategias de simulación, ORA, un modelo innovador para aprender del medio (pp. 9- 17). Octaedro Universidad.

Morgado, A. y Baena, J. (2011). Experimentación, Arqueología Experimental y experiencia del pasado en la Arqueología actual. En A. Morgado, J. Baena y D. García (Eds.), La investigación experimental aplicada a la arqueologia (pp. 21-27). Universidad de Granada.

Polo, A., García I. y Santos, E. (2014). La didáctica del patrimonio en la educación no formal y el tiempo libre en la ciudad de Segovia. En Actas de las IV Jornadas de Jóvenes investigadores del Valle del Duero (pp. 440-453). Glyphos.

Prats, J. y Santacana, J. (2011). Los restos arqueológicos, los monumentos y los museos como fuentes del pasado. En Geografia e historia: investigación, innovación y buenas prácticas (pp. 39-67). Secretaría General Técnica.

Quijano-Araníbar, I. E. (2018). El uso de la arqueología experimental como recurso didáctico en el proceso de aprendizaje: Una experiencia educativa en estudiantes de administración turística de Lima, Perú. Revista Electrónica Educare, 22(3), 287-313. https://doi.org/10.15359/ree.22-3.14

Ramos, M. L. (2007). El papel de la arqueología experimental en época romana y su didáctica. En M. Ramos, J. Urquijo y J. Baena (Eds.), Arqueología experimental en la Peninsula Ibérica: investigación, didáctica y patrimonio (pp. 9-20). Asociación Española de Arqueología Experimental. 
Schadla-Hall, T. (1999). Editorial: Public Archaeology. European Journal of Archaeology, 2(2), 147-158. https://doi.org/10.1179/eja.1999.2.2.147

Sáiz, J. y Fuster, C. (2014). Memorizar historia sin aprender pensamiento histórico. Las PAU de Historia de España. Investigación en la Escuela, 84, 47-58.

Sanmartí, N. (2010). Evaluar para aprender. Graó.

Santacana, J. (2005). Reflexiones en torno al laboratorio escolar en ciencias sociales. Iber: Didáctica de las ciencias sociales, geografía e historia, 43, 7-14.

Santacana, J. (2018). La arqueología y el reto de educar. En A. Egea, L. Arias y J. Santacana (Eds.), Y la arqueología llegó al aula. La cultura material y el método arqueológico para la enseñanza de la historia y el patrimonio (pp. 9-21). Trea.

Santacana, J. y Hernández, F. (1999). Enseñanza de la arqueología y la prehistoria: problemas y métodos. Milenio.

Santacana, J., López, V. y Martínez, T. (Coords.) (2017). La ciencia que no se aprende en la Red. Modelos didácticos para motivar el estudio de las ciencias a través de la arqueología. Graó.

Seel, N. M. (2008). Empirical perspectives on memory and motivation. En J. M. Spector, M. D. Merrill, J. Van Merriënboer \& M. P. Driscoll (Eds.), Handbook of research on educational communications and technology (3rd ed, pp. 39-54). Lawrence Erlbaum Associates.

Villanueva, G., Payno, M. E. y Fernández, L. (2016). Una propuesta didáctica: El tratamiento de pieles. útiles, elaboración y difusión. Boletín de Arqueología Experimental, 11, 150-167.

Wearing, J. (2011). Teaching archaeological thinking: A professional resource to help teach six interrelated concepts central to students' ability to think critically about archaeology. Critical Thinking Consortium.

Zabala, A. y Arnau, L. (2014). Métodos para la enseñanza de las competencias. Graó. 